九州大学学術情報リポジトリ

Kyushu University Institutional Repository

\title{
血液透析患者における心胸郭比と総死亡および心血 管疾患イベント：Qコホート研究
}

四枝，龍佑

http://hdl. hand le. net/2324/2236108

出版情報：Kyushu University，2018，博士（医学），課程博士 バージョン：

権利関係 : 


\section{Cardiothoracic Ratio and All-Cause Mortality and Cardiovascular Disease Events in Hemodialysis Patients: The Q-Cohort Study}

Ryusuke Yotsueda, MD, ${ }^{1}$ Masatomo Taniguchi, $M D, P h D,{ }^{2}$

Shigeru Tanaka, MD, PhD, ${ }^{1,3}$ Masahiro Eriguchi, MD, PhD, ${ }^{1}$ Kiichiro Fujisaki, MD, PhD, ${ }^{1}$ Kumiko Torisu, MD, PhD, ${ }^{1}$ Kosuke Masutani, MD, PhD, ${ }^{1}$ Hideki Hirakata, MD, PhD, ${ }^{2}$ Takanari Kitazono, MD, PhD, ${ }^{1}$ and Kazuhiko Tsuruya, MD, $P h D^{1,4}$

\footnotetext{
Background: Cardiothoracic ratio by chest radiography is commonly used to assess volume status. Little is known about the relationships between cardiothoracic ratio and the incidence of clinical outcomes in patients undergoing hemodialysis (HD).

Study Design: Prospective cohort study.

Setting \& Participants: 3,436 participants in the Q-Cohort Study 18 years or older who underwent maintenance HD in Japan.

Predictor: Cardiothoracic ratio.

Outcomes \& Measurements: All-cause mortality and cardiovascular disease (CVD) events.

Results: During a 4-year follow-up period, 564 (16.4\%) patients died of any cause and 590 (17.2\%) developed CVD events. From baseline cardiothoracic ratios, participants were categorized into sex-specific quartiles because cardiothoracic ratio distribution differed by sex. The 4-year event-free survival rate, in terms of all-cause mortality and CVD events, was significantly lower with higher cardiothoracic ratios. Compared to the lowest cardiothoracic ratio (quartile 1), multivariable-adjusted HRs for all-cause mortality were 0.89 (95\% Cl, 0.66-1.20), 1.41 (1.08-1.86), and 1.52 (1.17-2.00) in patients from quartiles 2, 3, and 4, respectively. Similarly, in comparison to quartile 1, multivariable-adjusted HRs for CVD events were 1.00 $(95 \% \mathrm{Cl}, 0.77-1.31), 1.18(0.92-1.53)$, and $1.37(1.07-1.76)$ in patients from quartiles 2,3 , and 4 , respectively. Furthermore, the combination of higher cardiothoracic ratio and normohypotension (systolic blood pressure $<140 \mathrm{~mm} \mathrm{Hg}$ and diastolic blood pressure $<90 \mathrm{~mm} \mathrm{Hg}$ ) was associated with higher risk for CVD events.

Limitations: Single measurement of all variables, potentially less-heterogeneous patient population, and limited ascertainment of cardiac parameters and the outcomes.

Conclusions: Higher cardiothoracic ratio is associated with higher risk for both all-cause mortality and CVD events in patients undergoing $\mathrm{HD}$.

Am J Kidney Dis. 70(1):84-92. () 2017 by the National Kidney Foundation, Inc.

INDEX WORDS: Cardiothoracic ratio (CTR); cardiovascular disease (CVD); hemodialysis (HD); chest radiography; all-cause mortality; CVD event; volume status; volume overload; end-stage renal disease (ESRD); risk marker; prospective cohort.
}

$\mathbf{C}$ ardiovascular disease (CVD) is the main cause of death in patients undergoing dialysis. ${ }^{1}$ Cardiovascular death in this patient population is estimated to be 10 to 20 times higher than that of the

From the ${ }^{I}$ Department of Medicine and Clinical Science, Graduate School of Medical Sciences, Kyushu University; ${ }^{2}$ Fukuoka Renal Clinic; ${ }^{3}$ Division of Internal Medicine, Fukuoka Dental College; and ${ }^{4}$ Department of Integrated Therapy for Chronic Kidney Disease, Graduate School of Medical Sciences, Kyushu University, Fukuoka, Japan.

Received August 5, 2016. Accepted in revised form November 20, 2016. Originally published online February 10, 2017.

Address correspondence to Kazuhiko Tsuruya, MD, PhD, Department of Integrated Therapy for Chronic Kidney Disease, Graduate School of Medical Sciences, Kyushu University, 3-1-1 Maidashi, Higashi-ku, Fukuoka 812-8582, Japan. E-mail: tsuruya@intmed2.med.kyushu-u.ac.jp

(C) 2017 by the National Kidney Foundation, Inc. $0272-6386$

http://dx.doi.org/10.1053/j.ajkd.2016.11.026 general population. ${ }^{2,3}$ In Japan, up to $40 \%$ of deaths in patients undergoing hemodialysis (HD) are caused by heart failure, myocardial infarction, and stroke. ${ }^{4}$ Thus, estimation of risk for CVD events is a primary goal in improving the prognosis of patients undergoing maintenance HD.

Cardiothoracic ratio by chest radiography is a simple, cost-effective, and noninvasive examination for the assessment of volume status ${ }^{5}$ and is routinely measured in dialysis facilities. High cardiothoracic ratio has been correlated with high risk for mortality in populations undergoing dialysis, namely patients undergoing maintenance $\mathrm{HD},{ }^{6}$ patients with strict volume control treatment, ${ }^{7}$ patients without diabetes mellitus, ${ }^{8}$ and patients undergoing peritoneal dialysis.

Previous reports suggest that high cardiothoracic ratio is an independent risk factor for cardiac dysfunction and left ventricular hypertrophy in hypertensive patients. ${ }^{10}$ Volume overload in patients 
undergoing dialysis is strongly connected to the development of hypertension, a significant risk factor for CVD. ${ }^{11}$ Cardiothoracic ratio could potentially be a convenient means for detecting uncontrolled hypertension and excessive volume overload and hence presumably could be useful as a risk marker for CVD. However, only a limited number of short-term, retrospective, ${ }^{6}$ or single-center ${ }^{7-9,12}$ studies have examined the relationships between cardiothoracic ratio and incidence of clinical outcomes (including all-cause mortality and CVD); the association therefore remains unclear. We conducted a large-scale prospective cohort study of patients undergoing HD to clarify the relationship between cardiothoracic ratio and both all-cause mortality and incidence of CVD.

\section{METHODS}

\section{Study Population}

The Q-Cohort Study is a prospective, longitudinal, multicenter, observational study in HD patients. A total of 3,598 outpatients 18 years or older who underwent HD at 39 dialysis facilities in Fukuoka and Saga prefectures in Kyushu, Japan, were eligible to participate in the study. Patients were enrolled from December 2006 to December 2007. Patients without demographic data $(\mathrm{n}=65)$ and patients for whom clinical outcome was not available $(n=97)$ were excluded. The remaining 3,436 patients were enrolled in the study. The study was conducted with approval from the Kyushu University Institutional Review Board for Clinical Research (approval number 20-31). Written informed consent was obtained from all patients. The study was registered in the University Hospital Medical Information Network (UMIN) clinical trial registry (UMIN ID: 000000556) and was performed according to the Ethics of Clinical Research (Declaration of Helsinki) requirements.

\section{Follow-up}

Patients were followed up from the date of their study registration to December 2010. Their health conditions were checked annually by physicians at each dialysis facility and by mail or telephone for patients who moved to other dialysis facilities not participating in the study.

\section{Calculation of Cardiothoracic Ratio}

Cardiothoracic ratio was calculated from a standard chest radiograph. The maximal horizontal diameter of the heart was divided by the horizontal inner width of the rib cage and then expressed as a percentage. Chest radiographs were performed by following the policy of each dialysis facility. In almost all dialysis facilities, chest radiographs were routinely performed predialysis after a 2-day interdialytic interval in an upright posterior-anterior view, as recommended in the Japanese guideline. ${ }^{13} \mathrm{We}$ measured cardiothoracic ratio at the beginning of the observation period.

\section{Outcomes}

The primary outcome was incidence of death from any cause. The secondary outcome was major cardiovascular events, which were defined as first-ever development of cardiovascular death, stroke, myocardial infarction, hospitalization for unstable angina, coronary intervention (coronary artery bypass surgery or angioplasty), hospitalization for heart failure, and peripheral artery disease. Each disease was defined as previously described. ${ }^{14-16}$ Briefly, stroke was defined as sudden onset of a nonocclusive and focal neurologic deficit persisting for more than 24 hours. Myocardial infarction was defined as a diagnosis based on prolonged severe chest pain, elevated levels of abnormal cardiac biochemical parameters, diagnostic electrocardiographic changes, and morphologic changes (including local asynergy of cardiac wall motion on electrocardiography or a persistent perfusion defect on cardiac scintigraphy). Unstable angina was defined as a medical condition involving chest pain, abnormally elevated levels of cardiac biomarkers, and diagnostic electrocardiographic changes that did not meet criteria for myocardial infarction. Heart failure was defined as an unplanned presentation to an acute-care setting with signs and symptoms that required active treatment for fluid removal. Peripheral artery disease was defined as tissue necrosis, lower-limb amputation, and revascularization procedure for the peripheral artery. All events were collected from patients' medical records.

\section{Risk Factor Measurements}

The details of risk factor measurements were previously published. ${ }^{14-16}$ Briefly, demographic information (eg, age, sex, HD vintage, and cause of end-stage kidney disease [diabetic nephropathy or other]) and clinical data (eg, hemoglobin, serum albumin, serum C-reactive protein [CRP], systolic blood pressure $[\mathrm{BP}]$, diastolic $\mathrm{BP}$, body mass index [BMI], and $\mathrm{Kt} / \mathrm{V}$ ) were collected at baseline. Physicians at each dialysis facility reported on the use of antihypertensive drugs, such as angiotensinconverting enzyme inhibitors and angiotensin II receptor blockers, and a history of diabetes or CVD. History of CVD includes cerebrovascular disease, coronary artery disease, congestive heart failure, and peripheral vascular disease. Body height and weight were measured in light clothing without shoes. All available data for BP were measured predialysis and collected from the dialysis records. Blood samples were collected predialysis after a 2-day interdialytic interval from a vascular access. The day of blood sampling was not always the same as the day of a chest radiograph. Dialysis doses were measured by a single-pool Kt/V method.

\section{Statistical Analysis}

Baseline data, according to quartiles of cardiothoracic ratio, were presented as mean \pm standard deviation, median and interquartile range, or number and percentage. Distributions of baseline data stratified by quartiles of cardiothoracic ratio were compared using trend analysis. The event-free survival rate for all-cause mortality and CVD events according to cardiothoracic ratio was described by the Kaplan-Meier method and compared by log-rank test. Unadjusted and multivariable-adjusted hazard ratios (HRs) with 95\% confidence intervals (CIs) of all-cause mortality and CVD events according to cardiothoracic ratio were calculated using a Cox proportional hazards model. The assumption of the proportional hazards was checked graphically using the log cumulative hazard plots for each outcome according to cardiothoracic ratio. The multivariable-adjusted model was adjusted for age, sex, dialysis vintage, cause of end-stage kidney disease, history of CVD, antihypertensive agent use, systolic BP, serum albumin level, hemoglobin level, serum CRP level, BMI, and Kt/V. These variables were based on a priori clinical judgment and the existing investigations. Heterogeneity in the correlation between subgroups was tested by adding a multiplicative interaction term to the relevant Cox model. The high-cardiothoracic ratio group (quartiles 3 and 4) was compared with the low-cardiothoracic ratio group (quartiles 1 and 2) as the reference. Multivariable-adjusted associations of cardiothoracic ratio with HRs and $95 \%$ CIs for all-cause mortality and CVD events were plotted using restricted cubic spline. We used 4 knots located at the 5th, 35th, 65th, and 95th percentiles of cardiothoracic ratio as recommended. ${ }^{17}$ The multivariable-adjusted model was adjusted for age, sex, dialysis vintage, cause of 
end-stage kidney disease, history of CVD, antihypertensive agent use, systolic BP, serum albumin level, hemoglobin level, serum CRP level, BMI, and Kt/V. Age, dialysis vintage, systolic BP, serum albumin level, hemoglobin level, serum CRP level, BMI, and $\mathrm{Kt} / \mathrm{V}$ were treated as spline terms. The value of $50.0 \%$, which was the overall median cardiothoracic ratio value, was chosen as the reference for each spline plot. Statistical calculations were performed using JMP, version 11 for Windows (SAS Institute Inc), SAS software package, version 9.3 (SAS Institute Inc), and $\mathrm{R}$ statistical software, version 3.2.4 ( $\mathrm{R}$ Foundation for Statistical Computing). A difference was considered significant for $P<0.05$.

\section{RESULTS}

\section{Distributions of Cardiothoracic Ratio According to Sex}

Histograms of cardiothoracic ratios according to sex are shown in Fig 1 and show normal distributions for both male and female patients, but with different median cardiothoracic ratio values. Female patients (median value, 51.9\%) had higher cardiothoracic ratios than male patients (median value, 49.0\%).

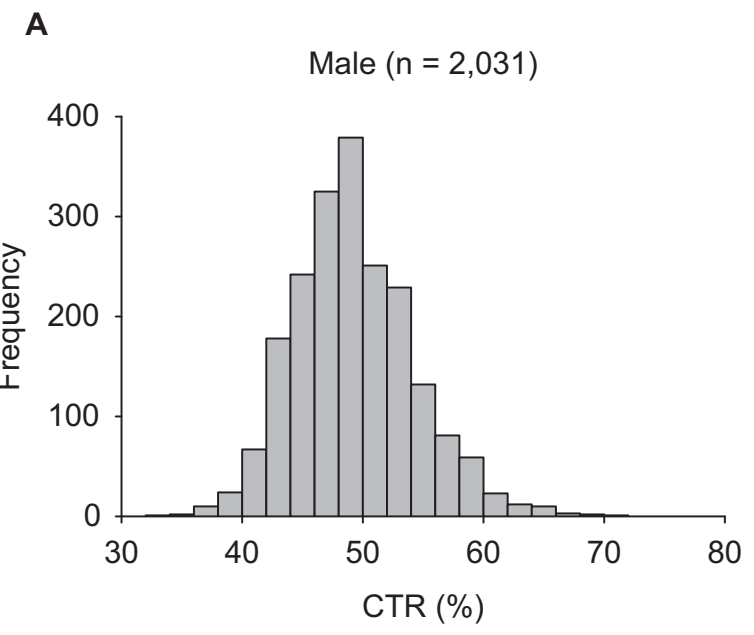

B

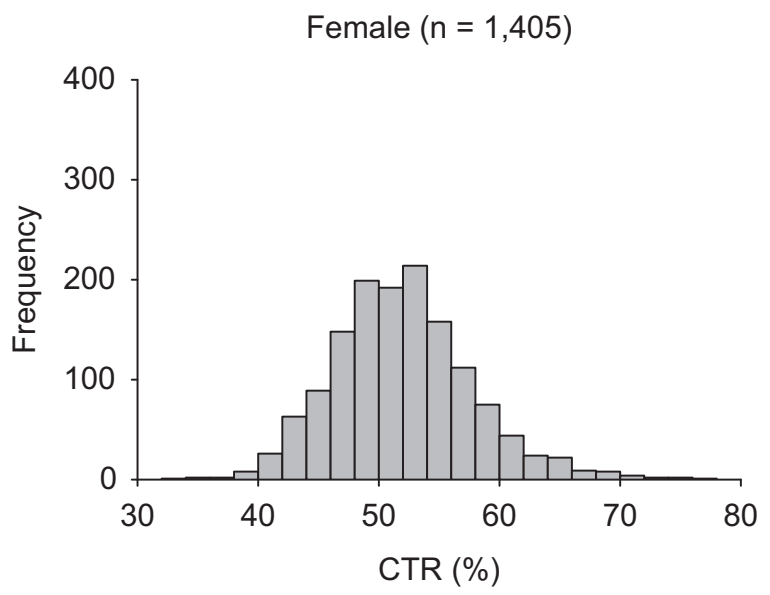

Figure 1. Histograms of cardiothoracic ratio (CTR) according to sex: (A) male and (B) female.
Quartiles of cardiothoracic ratio according to sex are shown in Table 1.

\section{Baseline Characteristics According to Sex-Specific Cardiothoracic Ratio Quartiles}

Baseline characteristics of patients based on sexspecific cardiothoracic ratio quartiles are listed in Table 1. Patients with higher cardiothoracic ratios were older and more likely to have a longer dialysis vintage; a history of CVD events, including brain infarction, brain hemorrhage, and heart disease; and a higher systolic BP. Median serum CRP levels also increased with higher cardiothoracic ratios. In contrast, mean values for serum albumin, hemoglobin, and $\mathrm{Kt} / \mathrm{V}$ decreased with higher cardiothoracic ratios.

\section{Association of Cardiothoracic Ratio With Risk for All-Cause Mortality and CVD Events}

\section{Categorical Analysis}

During the 4-year follow-up period, 564 (16.4\%) patients died of any cause (Table 2). Survival rates according to cardiothoracic ratio are shown in Fig 2A. The 4-year survival rate decreased with higher cardiothoracic ratios $(P<0.001)$. Patients with the highest cardiothoracic ratios (quartile 4) had a 1.52fold (95\% CI, 1.17- to 2.00-fold) higher risk for all-cause death than those with the lowest cardiothoracic ratio (quartile 1) after adjustment for potential confounders (Table 3). With regard to CVD events, during the 4-year follow-up period, $590(17.2 \%)$ patients developed CVD events (Table 2). Event-free survival rates according to cardiothoracic ratio are shown in Fig 2B. Higher cardiothoracic ratio was significantly associated with a lower event-free survival rate for CVD events $(P<0.001)$. The multivariable-adjusted risk for CVD events increased significantly, with 1.37 -fold (95\% CI, 1.07 - to 1.76 fold) higher risk in patients with the highest cardiothoracic ratios compared with patients with the lowest cardiothoracic ratios (Table 3).

\section{Continuous Analysis}

Multivariable-adjusted restricted cubic spline revealed that HRs for all-cause mortality and CVD events increased with increasing cardiothoracic ratios (Fig 3).

\section{Subgroup Analysis Stratified by Baseline Characteristics}

To assess the relationship between cardiothoracic ratio and patients' characteristics, the effect of modification of subgroups stratified by potential confounders was examined (Fig 4). A significant interaction was observed only between BP and CVD events. A clearer association between a high 
Table 1. Baseline Characteristics According to Sex-Specific Cardiothoracic Ratio Quartiles

\begin{tabular}{|c|c|c|c|c|c|c|}
\hline Variable & Total $(\mathrm{N}=\mathbf{3 , 4 3 6})$ & $\begin{array}{l}\text { Q1 }(n=837 \\
\text { M: }<46.0 \% \\
\text { F: }<48.3 \%)\end{array}$ & $\begin{array}{c}\text { Q2 }(n=848 \\
\text { M: } \mathbf{4 6 . 0 \% - < 4 9 . 0 \% ;} \\
\text { F: } 48.3 \%-<51.9 \%)\end{array}$ & $\begin{array}{c}\text { Q3 }(\mathbf{n}=\mathbf{8 8 6} \\
\text { M: } \mathbf{4 9 . 0 \% -}<\mathbf{5 2 . 5 \%} \\
\text { F: } \mathbf{5 1 . 9} \%-<55.5 \%)\end{array}$ & $\begin{array}{l}\text { Q4 (n = 865; } \\
M: \geq 52.5 \% \\
F: \geq 55.5 \%)\end{array}$ & $\begin{array}{l}P \text { for } \\
\text { Trend }\end{array}$ \\
\hline Age, y & $63.7 \pm 12.8$ & $58.4 \pm 13.1$ & $62.5 \pm 12.3$ & $64.7 \pm 11.9$ & $68.9 \pm 11.5$ & $<0.001$ \\
\hline Female sex & $1,405(40.9)$ & $351(41.9)$ & $346(40.8)$ & $354(40.0)$ & $354(40.9)$ & 0.6 \\
\hline Hemodialysis vintage, y & $5.5[2.1-11.5]$ & $4.4[1.8-10.4]$ & $5[1.9-10.9]$ & $6.1[2.4-12.2]$ & $6.8[2.8-12.7]$ & $<0.001$ \\
\hline Cause of ESKD: diabetes & $994(28.9)$ & 262 (31.3) & 240 (28.3) & $252(28.4)$ & $240(27.7)$ & 0.1 \\
\hline History of CVD & $1,158(33.7)$ & $184(22.0)$ & $229(27.0)$ & $310(35.0)$ & $435(50.3)$ & $<0.001$ \\
\hline History of brain infarction & 409 (11.9) & $78(9.3)$ & $74(8.7)$ & $104(11.7)$ & $153(17.7)$ & 0.002 \\
\hline History of brain hemorrhage & $126(3.7)$ & $17(2.0)$ & $32(3.8)$ & $31(3.5)$ & $46(5.3)$ & 0.004 \\
\hline History of heart disease & $775(22.6)$ & $104(12.4)$ & $144(17.0)$ & $218(24.6)$ & $309(35.7)$ & $<0.001$ \\
\hline Use of antihypertensive agent & $2,152(62.6)$ & $510(60.9)$ & $518(61.1)$ & $569(64.2)$ & $555(64.2)$ & 0.08 \\
\hline Use of ACE inhibitor/ARB & $1,489(43.3)$ & $370(44.2)$ & $366(43.2)$ & $382(43.1)$ & $371(42.9)$ & 0.6 \\
\hline Systolic BP, mm Hg & $153.0 \pm 23.4$ & $149.8 \pm 22.4$ & $151.1 \pm 22.9$ & $154.9 \pm 22.7$ & $156.1 \pm 25.1$ & $<0.001$ \\
\hline Diastolic BP, mm Hg & $76.4 \pm 12.3$ & $76.8 \pm 12.4$ & $76.0 \pm 12.0$ & $76.5 \pm 12.2$ & $76.4 \pm 13.8$ & 0.8 \\
\hline Serum albumin, g/dL & $3.8 \pm 0.4$ & $3.9 \pm 0.4$ & $3.8 \pm 0.4$ & $3.8 \pm 0.5$ & $3.7 \pm 0.5$ & $<0.001$ \\
\hline Hemoglobin, g/dL & $10.5 \pm 1.2$ & $10.6 \pm 1.1$ & $10.6 \pm 1.1$ & $10.5 \pm 1.1$ & $10.3 \pm 1.2$ & $<0.001$ \\
\hline Serum CRP, mg/dL & $0.13[0.06-0.30]$ & 0.10 [0.04-0.24] & $0.13[0.05-0.30]$ & $0.13[0.07-0.30]$ & $0.20[0.10-0.50]$ & $<0.001$ \\
\hline BMI, $\mathrm{kg} / \mathrm{m}^{2}$ & $21.1 \pm 3.1$ & $21.0 \pm 2.8$ & $21.3 \pm 2.9$ & $21.3 \pm 3.3$ & $21.0 \pm 3.4$ & 0.9 \\
\hline Single-pool Kt/V & $1.58 \pm 0.28$ & $1.60 \pm 0.27$ & $1.58 \pm 0.29$ & $1.57 \pm 0.27$ & $1.57 \pm 0.28$ & 0.02 \\
\hline
\end{tabular}

Note: Values for categorical variables are given as count (percentage); values for continuous variables, as mean \pm standard deviation or median [interquartile range].

Abbreviations: ACE, angiotensin-converting enzyme; ARB, angiotensin II receptor blocker; BMI, body mass index; BP, blood pressure; CRP, C-reactive protein; CVD, cardiovascular disease; ESKD, end-stage kidney disease; F, female; M, male; $Q$, quartile.

cardiothoracic ratio and a higher rate of CVD events was observed in patients with lower BPs than in those with higher BPs. Interactions were not evident for any other potential confounders.

\section{DISCUSSION}

In this prospective cohort study of patients undergoing HD, we clearly demonstrated that high cardiothoracic ratio was associated with high risk for all-cause mortality and CVD events. Additionally, subgroup analysis showed that the higher risk for CVD events was more significant in patients with normotension/hypotension than in those with hypertension. We highlighted the usefulness of cardiothoracic ratio as a potential risk marker for death and
CVD in patients undergoing maintenance HD. Furthermore, findings from our stratified analysis emphasized that the clinical significance of high cardiothoracic ratio might be affected by background comorbid conditions.

In the present study, high cardiothoracic ratio was independently associated with high risk for all-cause mortality and CVD events. These associations did not change even after adjustment for the potential confounders of age, sex, dialysis vintage, cause of end-stage kidney disease, history of CVD, antihypertensive agent use, systolic BP, serum albumin level, hemoglobin level, serum CRP level, BMI, and $\mathrm{Kt} / \mathrm{V}$. High cardiothoracic ratio has been correlated with high risk for mortality in a population

Table 2. Numbers of Individual Cardiovascular Disease Events by Sex in Each Cardiothoracic Ratio Quartile

\begin{tabular}{|c|c|c|c|c|c|c|c|c|c|}
\hline & \multirow[b]{2}{*}{ Total } & \multicolumn{2}{|c|}{ Q1 } & \multicolumn{2}{|c|}{ Q2 } & \multicolumn{2}{|c|}{ Q3 } & \multicolumn{2}{|c|}{ Q4 } \\
\hline & & $\begin{array}{c}\text { M: } \\
<46.0 \%\end{array}$ & $\begin{array}{c}\mathrm{F}: \\
<\mathbf{4 8 . 3} \%\end{array}$ & $\begin{array}{c}\text { M: } \\
46.0 \%-<49.0 \%\end{array}$ & $\begin{array}{c}\mathrm{F}: \\
48.3 \%-<51.9 \%\end{array}$ & $\begin{array}{c}\text { M: } \\
49.0 \%-<52.5 \%\end{array}$ & $\begin{array}{c}\mathrm{F}: \\
51.9 \%-<55.5 \%\end{array}$ & $\begin{array}{c}\mathrm{M}: \\
\geq \mathbf{5 2 . 5 \%}\end{array}$ & $\begin{array}{c}\mathrm{F}: \\
\geq \mathbf{5 5 . 5} \%\end{array}$ \\
\hline No. of patients & 3,436 & 486 & 351 & 502 & 346 & 532 & 354 & 511 & 354 \\
\hline No. of deaths & $564(16.4)$ & $53(10.9)$ & $28(8.0)$ & $58(11.6)$ & $33(9.5)$ & $93(17.5)$ & $61(17.2)$ & $144(28.2)$ & $94(26.6)$ \\
\hline \multicolumn{10}{|l|}{ No. of CVD events } \\
\hline All CVD events & 590 (17.2) & $72(14.8)$ & $35(10.0)$ & $86(17.1)$ & $35(10.1)$ & 102 (19.2) & $53(15.0)$ & $126(24.7)$ & 81 (22.9) \\
\hline Brain infarction & $141(4.1)$ & 16 (3.3) & $10(2.8)$ & $26(5.2)$ & $6(1.7)$ & $13(2.4)$ & $13(3.7)$ & $29(5.7)$ & $28(7.9)$ \\
\hline Brain hemorrhage & $77(2.2)$ & $8(1.6)$ & $3(0.9)$ & $18(3.6)$ & $3(0.9)$ & $17(3.2)$ & $5(1.4)$ & $12(2.3)$ & $11(3.1)$ \\
\hline Other & $372(10.8)$ & $48(9.9)$ & $22(6.3)$ & $42(8.4)$ & $26(7.5)$ & $72(13.5)$ & $35(9.9)$ & 85 (16.6) & 42 (11.9) \\
\hline
\end{tabular}

Note: Values are given as number and number (percentage).

Abbreviations: CVD, cardiovascular disease; F, female; M, male; $Q$, quartile. 
A

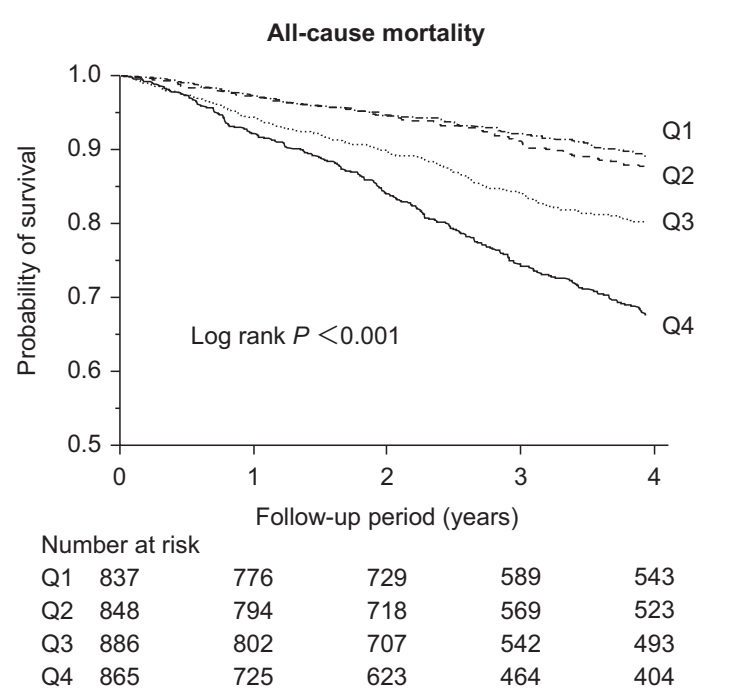

B

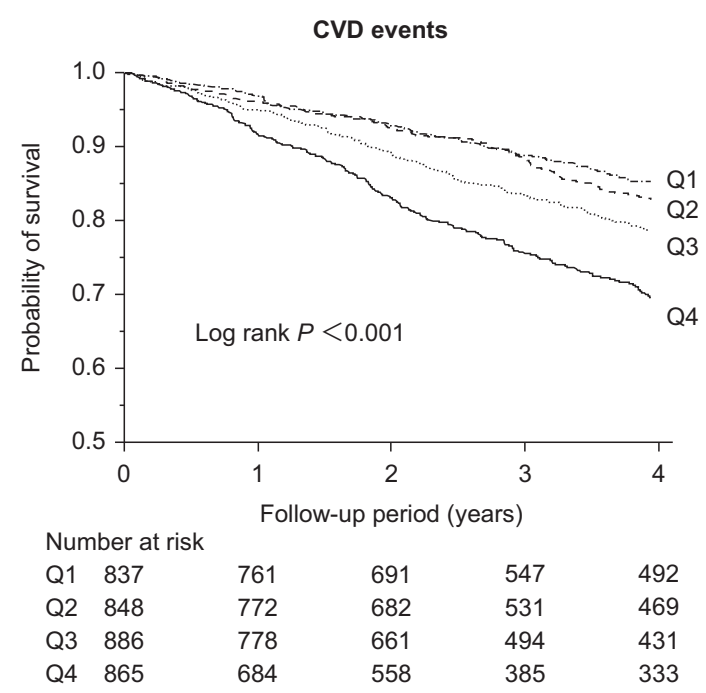

Figure 2. Event-free survival rate for (A) all-cause mortality and (B) cardiovascular disease (CVD) events according to sex-specific cardiothoracic ratio quartiles $(Q)$ during the 4-year follow up period.

undergoing dialysis. ${ }^{6-9}$ A report from Turkey demonstrated that the 5-year mortality of HD patients with cardiothoracic ratios $\geq 48 \%$ was higher than that of patients with cardiothoracic ratios $<48 \%$. $^{7}$ Our results were consistent with these useful clinical findings.

Several mechanisms might explain the increased risk for mortality and CVD events in patients with higher cardiothoracic ratios. First, cardiothoracic ratio is recognized as a sensitive marker for detecting volume overload. Volume overload is known as a factor that exacerbates arteriosclerosis through reactive oxygen species-related mechanisms, such as inappropriate activation of the renin-angiotensin-aldosterone system $^{18-21}$ and impaired nitric oxide-mediated vascular endothelial dysfunction ${ }^{22-25}$ arising from excessive pressure injury. Therefore, we presume that high cardiothoracic ratio might reflect a greater arteriosclerotic burden caused by volume overload. Second, high cardiothoracic ratio also might reflect a low state of cardiac function caused by potential or overt congestive heart failure. ${ }^{26}$ Unfortunately, we did not obtain detailed information about the diagnosis and severity of heart failure using atrial/brain natriuretic peptide levels, echocardiography, and New York Heart Association functional classification. Further studies should therefore be performed to investigate these indicators.

Table 3. Hazard Ratios for All-cause Death and CVD Events by Sex-Specific Cardiothoracic Ratio Quartiles

\begin{tabular}{|c|c|c|c|c|c|}
\hline & $\begin{array}{c}\text { Q2 (M: } \mathbf{4 6 . 0} \%-<49.0 \% \text {; } \\
\text { F: } \mathbf{4 8 . 3} \%-<\mathbf{5 1 . 9} \% \text { ) }\end{array}$ & $\begin{array}{c}\text { Q3 (M: } \mathbf{4 9 . 0} \%-<52.5 \% \text {; } \\
\text { F: } 51.9 \%-<55.5 \%)\end{array}$ & $\begin{array}{l}\text { Q4 (M: } \geq 52.5 \% \\
\text { F: } \geq 55.5 \%)\end{array}$ & $\begin{array}{c}\text { Every } 1 \% \\
\text { Increase in CTR }\end{array}$ & $\begin{array}{c}\text { Every } 1-\mathrm{SD}^{\mathrm{a}} \\
\text { Increase in CTR }\end{array}$ \\
\hline \multicolumn{6}{|l|}{ All-cause death } \\
\hline Unadjusted HR & $1.14(0.85-1.54)$ & $1.96(1.50-2.57)$ & $3.38(2.64-4.37)$ & $1.08(1.07-1.10)$ & $1.55(1.44-1.67)$ \\
\hline Age- and sex-adjusted HR & $0.92(0.68-1.25)$ & $1.47(1.12-1.93)$ & $2.00(1.55-2.60)$ & $1.06(1.04-1.07)$ & $1.37(1.26-1.48)$ \\
\hline Multivariable-adjusted HR & $0.89(0.66-1.20)$ & $1.41(1.08-1.86)$ & $1.52(1.17-2.00)$ & $1.04(1.02-1.05)$ & $1.22(1.12-1.33)$ \\
\hline \multicolumn{6}{|l|}{ CVD events } \\
\hline Unadjusted HR & $1.15(0.89-1.49)$ & $1.51(1.18-1.93)$ & $2.31(1.83-2.92)$ & $1.06(1.04-1.07)$ & $1.35(1.25-1.45)$ \\
\hline Age- and sex-adjusted HR & $1.01(0.78-1.31)$ & $1.26(0.98-1.62)$ & $1.70(1.34-2.16)$ & $1.05(1.03-1.06)$ & $1.29(1.19-1.41)$ \\
\hline Multivariable-adjusted HR & $1.00(0.77-1.31)$ & $1.18(0.92-1.53)$ & $1.37(1.07-1.76)$ & $1.03(1.01-1.05)$ & $1.18(1.08-1.29)$ \\
\hline
\end{tabular}

Note: Values are given as HR (95\% confidence interval). Quartile 1 (M: $<46.0 \% ; \mathrm{F}:<48.3 \%)$ was the reference category. The multivariable-adjusted model is adjusted for age, sex, dialysis vintage, cause of end-stage kidney disease, history of CVD, antihypertensive agent use, systolic blood pressure, serum albumin level, hemoglobin level, serum C-reactive protein level, body mass index, and $\mathrm{Kt} / \mathrm{V}$.

Abbreviations: CTR, cardiothoracic ratio; CVD, cardiovascular disease; F, female; HR, hazard ratio; M, male; Q, quartile; SD, standard deviation.

$\mathrm{a}_{1} \mathrm{SD}$ of CTR was $5.5 \%$. 


\section{A}

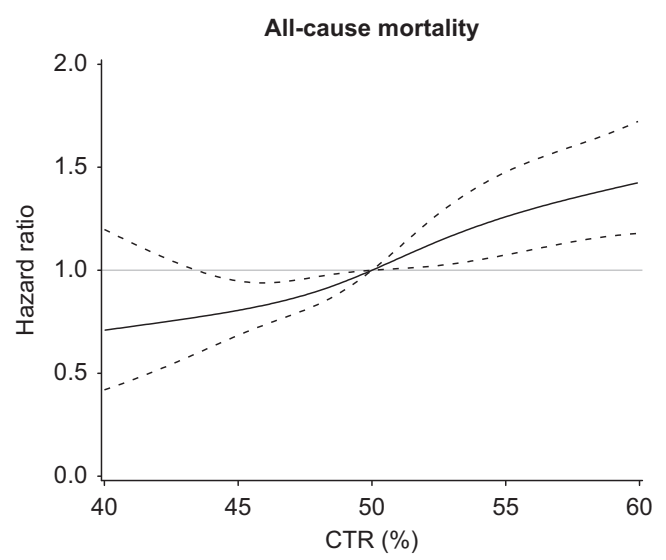

B

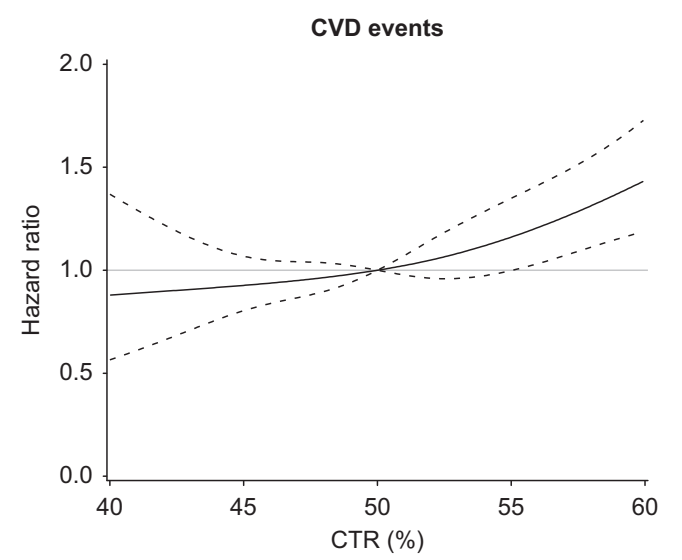

Figure 3. Multivariable-adjusted restricted cubic spline plots of hazard ratio (HR) for (A) all-cause mortality or (B) cardiovascular disease (CVD) events according to cardiothoracic ratio (CTR). Solid line represents HR and dotted line represents $95 \%$ confidence interval. The horizontal gray line corresponds to the normal reference HR of 1.0 . The overall median value of CTR was $50.0 \%$ and was chosen as the reference. The multivariable-adjusted model was adjusted for age, sex, dialysis vintage, cause of end-stage kidney disease, history of CVD, antihypertensive agent use, systolic blood pressure, serum albumin level, hemoglobin level, serum C-reactive protein level, body mass index, and $\mathrm{Kt} / \mathrm{V}$.

In subgroup analysis stratified by baseline characteristics, only an interaction between cardiothoracic ratio and BP on CVD events was evident. The correlation between high cardiothoracic ratio and high risk for CVD events was more remarkable in patients with normo-hypotension than in those with hypertension. Several lines of evidence are consistent with our results. Some studies have revealed the existence of a J-or U-shaped association between mortality and $\mathrm{BP}$ in patients undergoing $\mathrm{HD}^{27-29} \mathrm{~A}$ possible explanation of the inverse relationship between mortality and BP is that hypotension with severe heart failure and low cardiac function might worsen patient prognosis. ${ }^{27}$ Hence, it seems likely that the combination of high cardiothoracic ratio and low BP should be regarded as a reflection of low cardiac function attributable to severe heart failure.

The strength of our study is its wide-ranging nonrestrictive inclusion criteria. Only a few previous reports to date have examined the relationship between cardiothoracic ratio and clinical events in dialysis patients. These studies were conducted in limited populations, such as patients under strict volume control without antihypertensive drugs, ${ }^{7}$ without diabetes mellitus, ${ }^{8}$ and undergoing peritoneal dialysis. ${ }^{9}$ Therefore, these previous findings may not extrapolate to other populations. Our results could potentially be more generalizable to the population undergoing HD because of our wide-ranging inclusion criteria. Another strength of our study is its prospective cohort study design, on a larger scale and with longer follow-up than previous reports. ${ }^{6}$ We believe that our results offer useful clinical information toward understanding the association between cardiothoracic ratio and mortality and CVD events.

There were several limitations in our study. First, cardiothoracic ratio data were collected at only the baseline examination; thus, misclassification of participants into the different categories may have arisen. If this misclassification occurred, it would weaken the associations revealed in our study, biasing results toward a null hypothesis. Second, participating patients in our cohort study might not be fully representative of the overall Japanese HD population. Because participating facilities might reflect their clinical practice patterns, the management of patients could potentially be less heterogeneous than in other regions of Japan. Third, measurement of baseline parameters was not comprehensive. In particular, cardiac parameters, such as history of individual heart disease, atrial fibrillation, and use of anticoagulants and antiplatelet agents, were missing. Fourth, the available breakdown of CVD events was not detailed (Table 2). We could not evaluate definite CVD events excluding simple fluid overload, which was potentially included as heart failure. Further studies are required to determine these limitations, which could not be addressed because of our study design.

In conclusion, our results reveal that a high cardiothoracic ratio is associated with high risk for all-cause mortality and CVD events. Moreover, our findings suggest that the clinical significance of a high cardiothoracic ratio may be affected by patients' comorbid conditions. However, further 
A

\begin{tabular}{|c|c|c|}
\hline Variable & $\begin{array}{l}\text { No. of } \\
\text { Events }\end{array}$ & $\begin{array}{c}\text { No. of } \\
\text { Patients }\end{array}$ \\
\hline \multicolumn{3}{|l|}{ Sex } \\
\hline Female & 216 & 1,405 \\
\hline Male & 348 & 2,031 \\
\hline \multicolumn{3}{|l|}{ Age } \\
\hline Younger (<65) & 134 & 1,779 \\
\hline Older $(\geq 65)$ & 430 & 1,657 \\
\hline \multicolumn{3}{|l|}{ Etiology of endstage kidney disease } \\
\hline Non-diabetic nephropathy & 371 & 2,442 \\
\hline Diabetic nephropathy & 193 & 994 \\
\hline \multicolumn{3}{|l|}{ Past history of CVD } \\
\hline Absence & 253 & 2,278 \\
\hline Presence & 311 & 1,158 \\
\hline \multicolumn{3}{|l|}{ Blood pressure } \\
\hline Lower & 176 & 887 \\
\hline Higher & 388 & 2,549 \\
\hline
\end{tabular}

B

\begin{tabular}{|c|c|c|}
\hline Variable & $\begin{array}{l}\text { No. of } \\
\text { Events }\end{array}$ & $\begin{array}{l}\text { No. of } \\
\text { Patients }\end{array}$ \\
\hline \multicolumn{3}{|l|}{ Sex } \\
\hline Female & 204 & 1,405 \\
\hline Male & 386 & 2,031 \\
\hline \multicolumn{3}{|l|}{ Age } \\
\hline Younger $(<65)$ & 217 & 1,779 \\
\hline Older $(\geq 65)$ & 373 & 1,657 \\
\hline \multicolumn{3}{|l|}{ Etiology of endstage kidney disease } \\
\hline Non-diabetic nephropathy & 346 & 2,442 \\
\hline Diabetic nephropathy & 244 & 994 \\
\hline \multicolumn{3}{|l|}{ Past history of CVD } \\
\hline Absence & 258 & 2,278 \\
\hline Presence & 332 & 1,158 \\
\hline \multicolumn{3}{|l|}{ Blood pressure } \\
\hline Lower & 117 & 887 \\
\hline Higher & 473 & 2,549 \\
\hline
\end{tabular}

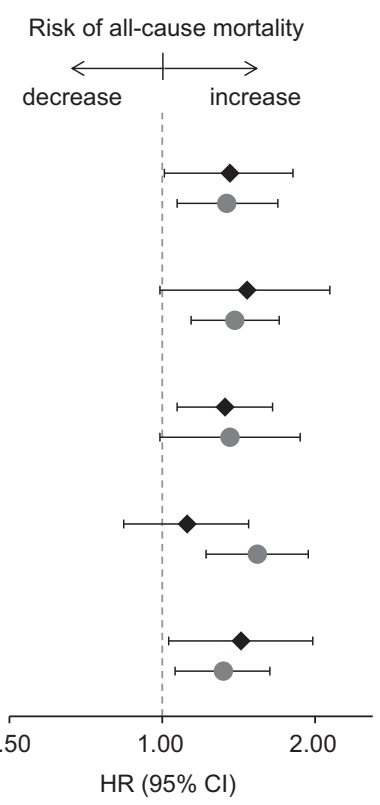

\begin{tabular}{lcc} 
& \multicolumn{2}{c}{$P$-value } \\
HR $(95 \% \mathrm{Cl})$ & effect & interaction \\
& & \\
$1.36(1.01-1.81)$ & 0.04 & 0.79 \\
$1.34(1.07-1.69)$ & 0.01 & \\
& & \\
$1.47(0.99-2.14)$ & 0.06 & 0.19 \\
$1.39(1.14-1.70)$ & 0.001 & \\
& & \\
$1.33(1.07-1.65)$ & 0.01 & 0.73 \\
$1.36(0.99-1.87)$ & 0.06 & \\
& & \\
$1.12(0.84-1.48)$ & 0.43 & 0.54 \\
$1.54(1.22-1.94)$ & 0.003 & \\
$1.43(1.03-1.98)$ & 0.03 & 0.17 \\
$1.32(1.06-1.63)$ & 0.01 & \\
& & \\
& &
\end{tabular}

Risk of CVD events

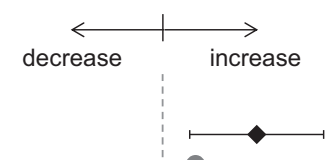

$\mathrm{HR}(95 \% \mathrm{Cl})$

$P$-value

effect interaction

$1.53(1.13-2.07) \quad 0.01$

$1.16(0.92-1.44) \quad 0.20$

0.14

$1.11(0.79-1.53) \quad 0.54$

$1.36(1.10-1.68) \quad 0.01$

0.42

$1.28(1.01-1.60)$

1.25 (0.93-1.66)

0.04

0.14

$1.39(1.04-1.85) \quad 0.03$

$1.19(0.95-1.49)$

0.13

0.43

$1.68(1.13-2.50)$

0.01

$1.19(0.97-1.45) \quad 0.09$

0.003

Figure 4. Multivariable-adjusted hazard ratios (HRs) and 95\% confidence intervals (Cls) for (A) all-cause mortality or (B) cardiovascular disease (CVD) events in higher cardiothoracic ratios according to subgroups of baseline characteristics. The high-cardiothoracic ratio group (quartiles 3 and 4) was compared with the low-cardiothoracic ratio group (quartiles 1 and 2) as the reference. The multivariable-adjusted model was adjusted for age, sex, dialysis vintage, cause of end-stage kidney disease, history of CVD, antihypertensive agent use, systolic blood pressure, serum albumin level, hemoglobin level, serum C-reactive protein level, body mass index, and $\mathrm{Kt} / \mathrm{V}$. Variables relevant to the subgroups were excluded from each model. Higher blood pressure was defined as systolic blood pressure $\geq 140 \mathrm{~mm} \mathrm{Hg}$ or diastolic blood pressure $\geq 90 \mathrm{~mm} \mathrm{Hg}$.

well-conducted prospective cohort studies or clinical trials are needed to determine the optimal range of cardiothoracic ratios. Additionally, whether practical approaches to reduce cardiothoracic ratio can improve the clinical prognosis of patients undergoing maintenance HD needs to be determined.

\section{ACKNOWLEDGEMENTS}

We thank participants in the Q-Cohort Study and members of the Society for the Study of Kidney Disease. The following personnel (institutions) participated in the study: Takashi Ando (Hakozaki Park Internal Medicine Clinic), Takashi Ariyoshi (Ariyoshi Clinic), Koichiro Goto (Goto Clinic), Fumitada Hattori 
(Nagao Hospital), Harumichi Higashi (St Mary's Hospital), Tadashi Hirano (Hakujyuji Hospital), Kei Hori (Munakata Medical Association Hospital), Takashi Inenaga (Ekisaikai Moji Hospital), Hidetoshi Kanai (Kokura Memorial Hospital), Shigemi Kiyama (Kiyama Naika), Tetsuo Komota (Komota Clinic), Hiromasa Kuma (Kuma Clinic), Toshiro Maeda (Kozenkai-Maeda Hospital), Junichi Makino (Makino Clinic), Dai Matsuo (Hirao Clinic), Chiaki Miishima (Miishima Clinic), Koji Mitsuiki (Japanese Red Cross Fukuoka Hospital), Kenichi Motomura (Motomura Naika Clinic), Sadatoshi Nakamura, Hidetoshi Nakamura (Kokura Daiichi Hospital), Koichi Nakashima (Ohashi Internal Circulatory Clinic), Nobumitsu Okita (Shiroishi Kyoritsu Hospital), Shinichiro Osato (Osato Jin Clinic), Sakura Sakamoto (Fujiyamato Spa Hospital), Keiko Shigematsu (Shigematsu Clinic), Kazumasa Shimamatsu (Shimamatsu Naika Iin), Yoshito Shogakiuchi (Shin-Ai Clinic), Hiroaki Takamura (Hara Hospital), Kazuhito Takeda (Iizuka Hospital), Asuka Terai (Chidoribashi Hospital), Hideyoshi Tanaka (Mojiko-Jin Clinic), Suguru Tomooka (Hakozaki Park Internal Medicine Clinic), Jiro Toyonaga (Fukuoka Renal Clinic), Hiroshi Tsuruta (Steel Memorial Yawata Hospital), Ryutaro Yamaguchi (Shiseikai Hospital), Taihei Yanagida (Saiseikai Yahata General Hospital), Tetsuro Yanase (Yanase Internal Medicine Clinic), Tetsuhiko Yoshida (Hamanomachi Hospital), Takahiro Yoshimitsu (Gofukumachi Kidney Clinic, Harasanshin Hospital), and Koji Yoshitomi (Yoshitomi Medical Clinic).

Support: This study was supported by the Kidney Foundation (H19 JKFB 07-13, H20 JKFB 08-8, H23 JKFB 11-11) and the Japan Dialysis Outcome Research Foundation (H19-076-02, H20003). The funders of this study had no role in study design; collection, analysis, and interpretation of data; writing the report; or the decision to submit the report for publication.

Financial Disclosure: The authors declare that they have no other relevant financial interests.

Contributions: Research idea and study design: RY, ST, ME, KTsuruya; data acquisition: MT, ST, ME, KF, KTorisu, KM, KTsuruya; data cleaning: KTsuruya; data interpretation: RY, ST, ME; statistical analysis: RY; supervision: HH, TK. Each author contributed important intellectual content during manuscript drafting or revision and accepts accountability for the overall work by ensuring that questions pertaining to the accuracy or integrity of any portion of the work are appropriately investigated and resolved. RY takes responsibility that this study has been reported honestly, accurately, and transparently; that no important aspects of the study have been omitted; and that any discrepancies from the study as planned have been explained.

Peer Review: Evaluated by 2 external peer reviewers, a Statistical Editor, a Co-Editor, and Editor-in-Chief Levey.

\section{REFERENCES}

1. Ortiz A, Covic A, Fliser D, et al. Epidemiology, contributors to, and clinical trials of mortality risk in chronic kidney failure. Lancet. 2014;383(9931):1831-1843.

2. Foley RN, Parfrey PS, Sarnak MJ. Clinical epidemiology of cardiovascular disease in chronic renal disease. Am J Kidney Dis. 1998;32(5)(suppl 3):S112-S119.

3. Foley RN, Parfrey PS, Sarnak MJ. Epidemiology of cardiovascular disease in chronic renal disease. J Am Soc Nephrol. 1998;9(12)(suppl):S16-S23.

4. Masakane I, Nakai S, Ogata S, et al. An overview of regular dialysis treatment in Japan (as of 31 December 2013). Ther Apher Dial. 2015;19(6):540-574.

5. Gunal AI. How to determine 'dry weight'? Kidney Int Suppl. 2013;3(4):377-379.
6. Bohn E, Tangri N, Gali B, et al. Predicting risk of mortality in dialysis patients: a retrospective cohort study evaluating the prognostic value of a simple chest X-ray. BMC Nephrol. 2013;14: 263.

7. Ozkahya M, Ok E, Toz H, et al. Long-term survival rates in haemodialysis patients treated with strict volume control. Nephrol Dial Transplant. 2006;21(12):3506-3513.

8. Chen KH, Lin-Tan DT, Huang WH, et al. Cardiothoracic ratio, malnutrition, inflammation, and two-year mortality in nondiabetic patients on maintenance hemodialysis. Kidney Blood Press Res. 2008;31(3):143-151.

9. Chen KH, Hung CC, Lin-Tan DT, et al. Cardiothoracic ratio association with mortality in patients on maintenance peritoneal dialysis. Ther Apher Dial. 2011;15(1):81-88.

10. Rayner BL, Goodman H, Opie LH. The chest radiograph. A useful investigation in the evaluation of hypertensive patients. Am J Hypertens. 2004;17(6):507-510.

11. Lawes CM, Vander Hoorn S, Rodgers A. Global burden of blood-pressure-related disease, 2001. Lancet. 2008;371(9623): 1513-1518.

12. Gao N, Kwan BC, Chow KM, et al. Longitudinal changes of cardiothoracic ratio and vascular pedicle width as predictors of volume status during one year in Chinese peritoneal dialysis patients. Kidney Blood Press Res. 2009;32(1):45-50.

13. Hirakata H, Nitta K, Inaba M, et al. Japanese Society for Dialysis Therapy guidelines for management of cardiovascular diseases in patients on chronic hemodialysis. Ther Apher Dial. 2012;16(5):387-435.

14. Eriguchi R, Taniguchi M, Ninomiya T, et al. Hyporesponsiveness to erythropoiesis-stimulating agent as a prognostic factor in Japanese hemodialysis patients: the Q-Cohort study. J Nephrol. 2015;28(2):217-225.

15. Tanaka S, Ninomiya T, Taniguchi M, et al. Comparison of oral versus intravenous vitamin $\mathrm{D}$ receptor activator in reducing infection-related mortality in hemodialysis patients: the Q-Cohort Study. Nephrol Dial Transplant. 2016;31(7):1152-1160.

16. Yamada S, Tsuruya K, Taniguchi M, et al. Association between serum phosphate levels and stroke risk in patients undergoing hemodialysis: the Q-Cohort Study. Stroke. 2016;47(9): 2189-2196.

17. Harrell FE. General aspects of fitting regression models. In: Regression Modeling Strategies: With Applications to Linear Models, Logistic Regression, and Survival Analysis. New York, NY: Springer New York; 2001:11-40.

18. K-Laflamme A, Oster L, Cardinal R, de Champlain J. Role of NO and angiotensin II in the early development of endothelial functions impairment and cardiac hypertrophy in deoxycorticosterone acetate-salt hypertension. Can J Physiol Pharmacol. 1998;76(6):665-675.

19. Usui M, Egashira K, Kitamoto S, et al. Pathogenic role of oxidative stress in vascular angiotensin-converting enzyme activation in long-term blockade of nitric oxide synthesis in rats. Hypertension. 1999;34(4, pt 1):546-551.

20. Poch E, Gonzalez D, Giner V, Bragulat E, Coca A, de La Sierra A. Molecular basis of salt sensitivity in human hypertension. Evaluation of renin-angiotensin-aldosterone system gene polymorphisms. Hypertension. 2001;38(5):1204-1209.

21. Reckelhoff JF, Romero JC. Role of oxidative stress in angiotensin-induced hypertension. Am J Physiol Regul Integr Comp Physiol. 2003;284(4):R893-R912.

22. Huang PL, Huang Z, Mashimo H, et al. Hypertension in mice lacking the gene for endothelial nitric oxide synthase. Nature. 1995;377(6546):239-242. 
23. Faraci FM, Sigmund CD, Shesely EG, Maeda N, Heistad DD. Responses of carotid artery in mice deficient in expression of the gene for endothelial NO synthase. Am J Physiol. 1998;274(2, pt 2):H564-H570.

24. Alvarez G, Osuna A, Wangensteen R, Vargas F. Interaction between nitric oxide and mineralocorticoids in the longterm control of blood pressure. Hypertension. 2000;35(3): $752-757$.

25. Kopkan L, Majid DS. Superoxide contributes to development of salt sensitivity and hypertension induced by nitric oxide deficiency. Hypertension. 2005;46(4):1026-1031.

26. Marantz PR, Tobin JN, Wassertheil-Smoller S, et al. The relationship between left ventricular systolic function and congestive heart failure diagnosed by clinical criteria. Circulation. 1988;77(3):607-612.

27. Foley RN, Parfrey PS, Harnett JD, Kent GM, Murray DC, Barre PE. Impact of hypertension on cardiomyopathy, morbidity and mortality in end-stage renal disease. Kidney Int. 1996;49(5): 1379-1385.

28. Zager PG, Nikolic J, Brown RH, et al. "U" curve association of blood pressure and mortality in hemodialysis patients. Medical Directors of Dialysis Clinic, Inc. Kidney Int. 1998;54(2): 561-569.

29. Port FK, Hulbert-Shearon TE, Wolfe RA, et al. Predialysis blood pressure and mortality risk in a national sample of maintenance hemodialysis patients. Am J Kidney Dis. 1999;33(3):507-517. 